\title{
Capturing the effect of a title on multiple levels of comprehension
}

\author{
Frances Daniel and Gary E. Raney \\ University of Illinois, Chicago, Illinois
}

\begin{abstract}
Researchers have manipulated text comprehension by creating texts that require a title to be understood, but the source of the comprehension deficit has not been fully examined. We created comprehension quizzes for these texts that measure the surface form, textbase, and situation model. In three experiments, participants read passages with or without a title and then answered quiz questions. Results showed that the absence of a title influenced the accuracy rate of answering situation model questions more than answering surface form or textbase questions. This suggests that the situation model is the primary source of difficulty for these texts. These passages and quizzes can be used in future research that requires controlled manipulation and measurement of situation level comprehension.
\end{abstract}

An important goal for reading research is to accurately measure what readers remember and comprehend after reading a text. van Dijk and Kinstch (1983) describe text comprehension and memory as comprising three levels: the surface form (lower-level comprehension, such as words), textbase (comprehension of sentences and phrases), and situation model (text-level understanding integrated with the reader's prior knowledge). Although these levels of processing have received much empirical support, isolating what is comprehended and remembered has proven to be very difficult. This is because readers can remember words (surface form) without understanding the gist (situation model), understand the gist but not remember the actual wording, or remember meaning units (textbase) but not understand the implications (situation model) (see Kintsch, Welsch, Schmalhofer, \& Zimny, 1990 , for a summary of supporting research).

The purpose of the present research is twofold: First, we describe a technique for manipulating situation model comprehension while maintaining comprehension of the surface form and textbase. Specifically, readers are presented with passages that allow situation model development when a title is provided but hinder situational development when a title is not provided. This technique has been used in several prior studies (Bransford \& Johnson, 1972; Levy, 2001; Wiley \& Rayner, 2000). Researchers generally assume that removing the title influences only the situation model, but, to our knowledge, this assumption has not been well validated. Our second purpose is to provide a set of passages and comprehension questions that can be used in future research. The questions were designed to measure comprehension and memory for the surface form, textbase, and situation model.

Several researchers have used rereading paradigms to explore factors that influence text comprehension (Levy, 2001; Levy et al., 1995; Raney, Therriault, \& Minkoff,
2000; see Raney, 2003, for review). A typical finding is that the second text is read faster than the first text. This reflects a repetition effect or transfer benefit. By changing features of a text between a first and second reading, it is possible to measure the impact of the changed feature on performance. For example, Raney et al. (2000) altered the surface form by changing key content words to synonyms during the second reading (e.g., swamp changed to marsh). This kept the textbase and situation model the same across readings. Others have manipulated the textbase by introducing new events and words. For example, Levy et al. (1995) altered the textbase but kept the situation model intact by having participants read successive chapters in the same book. There was surface form overlap because chapters shared content words. However, the meaning of phrases and sentences changed across chapters.

The situation model has proven to be more difficult to experimentally manipulate. This is because a complete situation model integrates the surface form and textbase with the reader's prior knowledge. One method for manipulating the situation model is to hinder the ability to create a situation model. Bransford and Johnson (1972) did this by creating passages that required a title for situation model development. When the title was removed, situation model comprehension was hindered because the topic was unclear. Reading time also increased when no title was provided. This finding has been referred to as the title effect (Levy, 2001).

Readers encounter processing difficulties when reading these special texts without a title (Bransford \& Johnson, 1972; Levy, 2001; Wiley \& Rayner, 2000). Processing difficulties are reflected in online measures, such as fixation patterns (Wiley \& Rayner, 2000) and reading times (Levy, 2001), and postprocessing measures, such as text recall (Bransford \& Johnson, 1972). Although it has been

F. Daniel, fdanie2@uic.edu; G. E. Raney, geraney@uic.edu 
suggested that the inability to create a situation model is the reason for processing difficulties, the situation model has never been isolated as the source of the difficulty. A related issue concerns passage development. Without proper norming to ensure proper stimuli development, researchers cannot be sure that the title manipulation only influences the situation model. The unique contribution of our research is that multiple levels of comprehension were measured after reading texts with and without titles to precisely determine how comprehension changes as a function of the presence/absence of a title.

We had several major objectives in mind when developing the questions. One objective was to develop questions that accurately measure surface level, textbase, and situation model knowledge of the texts. For each passage, we also had to ensure that each question referenced unique information within a passage, so that information within one question did not influence how another question was answered. The second objective was to verify that the title manipulation primarily influenced the accuracy of answering situation model questions. To do this, we also had to ensure that potential answers did not reveal the topic of the passage. If readers can answer situation model questions correctly without a title, then the title manipulation will not be effective. Likewise, if surface form or textbase knowledge is substantially altered by the removal of a title, then the source of processing difficulty cannot be just the situation model. Our third objective was to match questions on difficulty level across passages. This would allow comprehension to be compared across passages, which is necessary when comparing conditions within an experiment.

\section{Measuring Levels of Comprehension}

Surface level features of the text are considered shallow features, such as words. Readers can understand the words of a sentence without understanding the overall meaning of a passage.

The surface form questions used here measured memory for exact words. Participants were presented fouralternative multiple-choice questions. The question stem presented a sentence from the passage with a content word missing. The alternatives included the correct content word along with three synonyms. For example, after reading a text titled "Traditionally Mailing a Letter Versus E-mailing” (see Appendix A), participants were presented with the following question.

Further, it is on this basis that most individuals argue in favor of universally the latest approach.
(a) accepting
(b) securing
(c) acquiring
*(d) adopting

Choosing the correct answer (identified by *) indicates memory for wording. The multiple-choice method is similar to a cloze procedure, which requires readers to fill in the blank with the exact word used in the text. The multiple-choice method was used because chance performance (i.e., 25\%) can be easily determined.
The textbase refers to individual meaning units within a text, such as phrases or sentences. Although a title might influence how readers organize textual units, these units contain relatively similar meaning, regardless of whether a title is present. Therefore, the title should not substantially influence textbase construction. The title could serve as an organizational mechanism, which might aid memory of the textbase; however, the title should not drastically change the meaning of a textbase. The rate of correctly answering textbase questions should not change dramatically as a function of presence or absence of a title.

Multiple-choice questions have been successfully used to measure memory for the meaning units in the text (Dunlosky \& Rawson 2005; Haenggi \& Perfetti, 1992). For example, the following question measures memory for information about the "modern approach."

What is a disadvantage to the modern approach?

(a) It requires a fee each time the process is completed.

*(b) There is a required investment in expensive equipment.

(c) It is hard to find tools needed for this approach.

(d) This approach is less familiar to all people.

Choosing the correct answer demonstrates explicit understanding of the textbase.

Situation model understanding requires integration of the text with prior knowledge as well as integration of text features. One method for measuring situational understanding is asking the reader to apply information from the text to a novel situation. To do this, the reader must create a coherent representation that supports generalization. The nature of the passages used in this experiment required the presence of a title to create a coherent understanding of the text necessary for situational development. Therefore, the absence of a title should substantially decrease the rate of correctly answered situation model questions.

Questions that measure the situation model understanding typically require the reader to generate an inference based on the textual information or apply the textual information in a novel situation. Inferences that require readers to bridge gaps in the text using prior knowledge are associated with building situation models (McNamara, Kintsch, Songer, \& Kintsch, 1996), and the application of new information in a novel situation is considered to demonstrate deep learning, which is also dependent on the development of the situation model (Kintsch, 1994). Recall and multiple-choice questions are typically used to assess the development of inferences and knowledge application. One criticism of recall measures is that they do not ensure that the reader will provide inferences. Multiple-choice questions that measure the situation model solve this problem by requiring the reader to generate an inference to answer correctly. That is, the correct answer to the question is the inference the reader must generate to understand the passage on a global level. For example, choosing the correct answer to the following question illustrates that the reader understands the underlying concept of the modern 
method and is able to apply that concept beyond what the passage describes as its usage.

When using the traditional approach, what is required for each transaction?

(a) An investment in expensive equipment

*(b) An adhesive picture that is placed on the final product

(c) A line that indicates the content of the final product

(d) A push of a button that allows you to create the final product

Past research has successfully used inference and application questions to measure the situation model (Dunlosky \& Rawson, 2005; Haenggi \& Perfetti, 1992); therefore, multiple-choice questions were also used here.

As mentioned earlier, another objective was to match question difficulty across and within passages as much as possible, and, to the extent possible, to match the overall difficulty of surface form, textbase, and situation model questions. Because surface form, textbase, and situation model questions measure different types of knowledge, some differences in difficulty between question types are inevitable. Our goal was to minimize differences in difficulty across questions types.

\section{EXPERIMENT 1}

There were two primary purposes of Experiment 1 . The first purpose was to verify that the title manipulation mainly influenced situation model comprehension. The second purpose was to match question difficulty across and within passages to the extent possible.

\section{Method}

\section{Participants}

One-hundred and three undergraduates enrolled in introductory psychology courses at the University of Illinois at Chicago participated for course credit. Participants were randomly assigned to one of two conditions (described below). Participants were fluent in English, which was defined as speaking, writing, and reading in English in an educational setting for at least 10 years.

\section{Materials, Design, and Procedure}

Passages. Eight passages were taken from Levy (2001). These passages were modeled after the Bransford and Johnson (1972) texts that required a title for situation understanding. The content of these passages covered everyday activities, such as brushing your teeth and making coffee. Passages were about 400 words in length (range, 399-401) and contained an average of 25 sentences per passage (range, 21-29). Levy (2001) found the standard title effect for these passages (i.e., longer reading times when no title was provided), but comprehension was not tested. Our study isolates the source of the processing difficulty for these passages using the comprehension questions described next.

Comprehension questions. Twelve questions were developed for each passage ( 4 surface form, 4 textbase, and 4 situation model comprehension questions), for a total of 96 questions. The end goal was to develop 6 questions for each passage ( 2 surface form, 2 textbase, and 2 situaiton model), for a total of 48 questions. We started with a larger pool of questions to select those that best matched the objectives stated earlier.
Surface form questions were created by selecting sentences in the passage and removing a content word that was not the first or last word of a sentence. The correct word and three synonyms were presented as answers. Textbase questions were based on main concepts in the passage. The correct answer and three alternatives were presented as answers. Situation model questions were based on understanding the gist of the passage. These questions required the reader to apply the gist to a novel situation or make an inference based on the gist of the passage. The correct inference and three alternatives were presented as answers.

For all questions types, the potential answers did not contain cues about the topic but could be understood without a title. This ensured that the answers did not help the reader understand a passage when a title was absent. Surface form, textbase, and situation model questions did not overlap in content. That is, information presented in a surface form question did not overlap with information presented in a textbase question, which did not overlap with information presented in a situation model question. This minimized the possibility that answering one type of question would help the reader answer another type of question. This was necessary to isolate the source of comprehension difficulties when a title was not provided.

Procedure. Booklets were created that contained two sets of the eight passages (16 total questions). The order was as follows: A passage was presented on one page and the 6 comprehension questions ( 2 surface form, 2 textbase, and 2 situation model) on the next page. The following page contained a different passage, which was followed by a separate page for comprehension questions. This format occurred for all eight passages. After reading the passages once, the passages were repeated, but a new set of comprehension questions followed each passage (i.e., 2 new surface form, 2 new textbase, and 2 new situation model). Participants were not allowed to look back at the passages when answering questions.

Approximately half of the participants were shown titles (title, no look back; $n=50$ ) and half were not shown titles (no title, no look back; $n=53$ ). Titles appeared above the passages. Groups of 2-10 participated in the experiment at one time. Participants typically completed the experiment in $1-1.5 \mathrm{~h}$ but were allowed as much time needed. All conditions in this experiment and subsequent experiments were between participants.

\section{Results}

After tabulating accuracy rates for all 96 questions, 48 final questions were selected ( 2 surface form, 2 textbase, and 2 situation model for all eight passages) that best matched the objectives stated previously. In addition, we selected questions that did not elicit extremely high or low accuracy rates (those questions would have little diagnostic value). For situation model questions, accuracy was expected to approach chance levels when titles were not present. Appendix A contains one example text (with a title) along with all the questions for every text and the mean percent correct for each question.

Three paired $t$ tests were conducted to determine whether the presence/absence of a title influenced accuracy of answering the final set of 48 questions. Analyses were performed by participants $\left(t_{1}\right)$ and items $\left(t_{2}\right)$. The first set of $t$ tests demonstrated that there were no significant differences in accuracy when answering surface form questions with $(66 \%)$ and without $(62 \%)$ a title $\left[t_{1}(15)<2 ; t_{2}(7)<2\right]$. The second set of $t$ tests demonstrated that significantly more textbase questions were answered correctly with a title $(60 \%)$ than without a title $(50 \%)\left[t_{1}(15)=-4.40\right.$, $\left.p<.01 ; t_{2}(7)=4.21, p<.01\right]$. This small difference is not surprising, because the title can serve as an organiza- 
tional cue that can help readers remember the textbase. The last set of $t$ tests demonstrated that significantly more situation model questions were answered correctly with a title (71\%) than without a title (37\%) $\left[t_{1}(15)=5.73, p<\right.$ $\left..001 ; t_{2}(7)=15.95, p<.001\right]$. In summary, the presence/ absence of a title had a small influence on surface form (4\%) and textbase (10\%) comprehension, but a very large influence on situation model (34\%) comprehension. This validates the title manipulation and demonstrates that the primary source of comprehension difficulty when no title is presented is at the situation model level.

\section{EXPERIMENT 2}

The purpose of Experiment 2 was to test whether the reduction in accuracy for situation model questions when no title was present reflected memory effects as opposed to comprehension difficulty. As noted earlier, titles provide organizational cues. Thus, it is possible that the difference in accuracy with and without a title reflects changes in the ability to remember information, not differences in the ability to comprehend information. To test this possibility, in Experiment 2, participants were allowed to look back at the passages when answering questions, which should remove differences between conditions associated with the ability to remember the passage. A relatively small number of participants (30) could be used here because our only goal was to determine whether accuracy for situation model questions was equal with and without a title when participants could look back at the passage when answering questions. Approximately half of the participants were shown titles (title, look back; $n=14$ ) and half were not shown titles (no title, look back; $n=16$ ).

\section{Method}

Thirty undergraduates from the same population as in Experiment 1 participated. Experiment 2 used the same texts, design, and procedure as Experiment 1, except readers were allowed to look back at the passages while answering questions.

\section{Results}

To be consistent with Experiment 1, the entire set of 96 questions was given to participants in Experiment 2. For the 48 matched questions selected in Experiment 1 (see Appendix A), $t$ tests (by participants and items) revealed that the rate of answering surface form questions was not significantly different with $(90 \%)$ and without $(92 \%)$ a title $\left[t_{1}(15)<1 ; t_{2}(7)<1\right]$. The rate of correctly answering textbase questions was significantly greater with a title $(70 \%)$ than without a title $(56 \%)\left[t_{1}(15)=-3.16, p<\right.$ $\left..05 ; t_{2}(7)=4.32, p<.01\right]$. The rate of correctly answering situation model questions also was significantly greater with a title $(75 \%)$ than without a title $(38 \%)\left[t_{1}(15)=\right.$ $\left.-7.37, p<.001 ; t_{2}(7)=6.50, p<.001\right]$.

The pattern of results for Experiment 2 matched that in Experiment 1; there was no significant title effect for surface form questions (2\%) and a small title effect for textbase questions (14\%). More importantly, there was a large title effect for situation model questions (37\%). Relative to Experiment 1, accuracy in Experiment 2 increased for surface form and textbase questions when participants were allowed to look back at the passages. However, the rates of answering situation model questions correctly were similar in Experiments 1 and 2. This suggests that the difference in accuracy for situation model questions as a function of the presence/absence of a title does not result from limited cognitive resources or organizational cues. The difference results from the ability to develop a better situation model when the title is provided.

\section{EXPERIMENT 3}

Accuracy for situation model questions in Experiments 1 and 2 appeared to be slightly above pure chance $(25 \%)$. One explanation for this finding is that readers were able to eliminate some of the alternatives for some of the questions, which would increase the odds of answering correctly even if they guessed the final answers. Another explanation is that readers were able to derive cues about the topic from the questions and possible answers. This would be problematic because it would partly mitigate the effect of not providing a title and reduce the ability to isolate the source of comprehension difficulties. Experiment 3 was conducted to test the latter explanation and to replicate findings from Experiments 1 and 2 in a more controlled environment. This experiment used only the final set of 48 selected questions.

One way to test whether readers derive cues about the topic from the questions is through a rereading procedure. We had participants read the passages once and answer questions, then read the passages a second time and answer a new set of questions. If answering the first set of questions provided cues about the topic, then accuracy should substantially improve after the second reading. Rereading also tends to increase overall comprehension. Rereading has been shown to improve college students' ability to find spelling errors, which is a surface level error (Levy, Di Persio, \& Hollingshead, 1992), and their ability to recognize discontinuities in temporal, causal, and spatial aspects of texts, which reflects textbase and situation level errors (Zwaan, Magliano, \& Graesser, 1995). Withholding a title hinders situational development, in which case comprehension of the surface form and textbase might increase, whereas there should be little increase in situation model knowledge during the second reading.

To test these predictions, Experiment 3 was conducted using two conditions. In one condition, no titles were presented during the first and second readings (NT-NT). In the second condition, no titles were presented during the first readings, but they were during the second readings (NT-T). If readers derived the topic from the questions after the first reading, comprehension after the second reading of the NT-NT condition should match that in the NT-T condition, in which readers clearly would know the topic. Of course, an intermediate improvement is more likely. Adding a title during the second reading should produce a large increase in accuracy for situation model questions.

Comprehension was tested using only the final 48 questions selected in Experiment 1. This provides an opportunity to replicate the results from Experiments 1 and 2 with a smaller set of questions. It is possible that the large num- 
ber of questions used in Experiments 1 and 2 led readers to focus on comprehension and memory more than they would with a smaller number of questions. Participants were also tested individually to provide a more controlled experimental setting.

\section{Method}

\section{Participants}

Forty undergraduates from the same population used in Experiments 1 and 2 participated.

\section{Materials, Design, and Procedure}

Experiment 3 used the same texts as Experiments 1 and 2. Two additional texts were developed as fillers (not read twice). These texts were also designed to be easy to understand with a title but difficult to understand without a title. Participants only responded to the final set of 48 questions.

Each participant read the 10 passages once. After each passage, participants answered one surface form, one textbase, and one situation model question. They then read the eight target passages a second time in the same order as in the first reading (i.e., first and second readings were blocked). The two filler passages were read only once. These were the third and seventh passages read (out of 10). Millis, Simon, and tenBroek (1998) and Raney (1993) used this method to ensure that readers read for comprehension during first readings (i.e., readers expected to read some passages only once). After each second reading of a passage, participants answered three new questions (one surface form, one textbase, and one situation model). There were two comprehension quizzes for every passage (one after each reading), and the order in which each participant received the quizzes was chosen randomly.

Participants were told that they would read several passages on a computer and that they would read most of the passages twice. They were not told how many passages they would read twice. They were also told that they would take a quiz after each reading.

Passages were presented one sentence at a time on a computer monitor. The self-paced reading procedure was used because it is a common methodology. When reading a titled text, the title was presented before the first sentence appeared. All sentences were left justified and centered vertically. To advance from one sentence to the next, participants pressed the space bar. This caused the previous sentence to disappear and a new sentence to appear. After reading the last sentence of the passage, a message appeared indicating that the passage was finished and that questions would be presented.

Comprehension questions were randomly ordered and appeared one question at a time. Participants indicated their choice by pressing the letter key corresponding to the answer (A, B, C, or D). After completing the quiz, a warning screen appeared indicating that another passage would be read. Participants continued this cycle until all passages were read. After reading all the passages once, participants reread the eight target passages a second time and completed a second quiz with new questions. Participants practiced the computerized procedure by reading one practice passage before beginning the experiment.

\section{Results}

To test whether the accuracy rate after reading texts without a title exceeded chance, one-sample $t$ tests (by participants and items) were conducted using 2 (25\% accuracy) as the testing value. As shown in Table 1, for the NT-NT condition, accuracy rates for surface form and textbase questions significantly exceeded chance during the first reading [surface form, $t_{1}(19)=9.35, p<.001$; $t_{2}(7)=13.32, p<.001$; textbase, $t_{1}(19)=4.72, p<.01$; $\left.t_{2}(7)=4.18, p<.01\right]$. During the second reading, accuracy exceeded chance for surface form questions $\left[t_{1}(19)=\right.$ $\left.9.49, p<.001 ; t_{2}(7)=6.89, p<.001\right]$, textbase questions $\left[t_{1}(19)=5.73, p<.001 ; t_{2}(7)=3.58, p<.01\right]$, and situation model questions $\left[t_{1}(19)=3.33, p<.005 ; t_{2}(7)=\right.$ $2.61, p<.05]$. Although answering situation model questions at a level exceeding chance $(\mu=2.9)$ was not predicted, this was not entirely surprising. Because students had read the texts (even without a title), it was possible to gain some type of textual understanding that could have slightly aided in answering questions, or possibly eliminated an alternative for some questions, which would have allowed for above-chance performance. More important is that situation model questions clearly were answered less accurately than surface form and textbase questions after both the first and second readings, which suggests minimal situational representation. These results support the conclusion that the readers' mental representations contained good surface form and textbase knowledge but lacked well-developed situation-level knowledge.

The NT-T condition should not have supported creating a situational representation during the first reading but should have supported the development of a situation model during the second reading. Results support this prediction. As Table 1 shows, accuracy for the NT-T condition during the first reading was similar to NT-NT accuracy. Surface form and textbase questions were answered at a rate significantly greater than chance [surface form, $t_{1}(19)=10.43, p<.001 ; t_{2}(7)=7.32, p<.001$; textbase, $\left.t_{1}(19)=5.30, p<.005 ; t_{2}(7)=4.21, p<.005\right]$. On the other hand, situation model questions were not answered at a rate greater than chance $\left[t_{1}(19)<2 ; t_{2}(7)<1\right]$. After the second reading, all questions were answered at rates significantly greater than chance [surface form, $t_{1}(19)=$ $6.89, p<.001 ; t_{2}(7)=12.50, p<.001$; textbase, $t_{1}(19)=$ $11.17, p<.001 ; t_{2}(7)=5.81, p<.005$; situation model, $\left.t_{1}(19)=12.95, p<.001 ; t_{2}(7)=5.32, p<.005\right]$. With the introduction of a title, readers were able to add a situational component to their mental representations, which was reflected by the large increase in accuracy.

It could be argued that the NT-NT and NT-T conditions demonstrated similar comprehension patterns because in each condition, situation model questions were answered at greater-than-chance rates during the second reading. Examination of the means across question types argues against this conclusion. During the second reading of NT-NT, situation model questions were answered less accurately $(\mu=2.90)$ than surface form $(\mu=5.20)$

Table 1

Mean Number of Correct Question Responses (Comprehension) by Condition for First (R1) and Second (R2) Readings for Surface Form, Textbase, and Situation Model Questions for Experiment 3

\begin{tabular}{|c|c|c|c|c|c|c|c|c|}
\hline & \multicolumn{4}{|c|}{ NT-NT } & \multicolumn{4}{|c|}{ NT-T } \\
\hline & \multicolumn{2}{|c|}{$\mathrm{R} 1$} & \multicolumn{2}{|c|}{$\mathrm{R} 2$} & \multicolumn{2}{|c|}{$\mathrm{R} 1$} & \multicolumn{2}{|c|}{$\mathrm{R} 2$} \\
\hline & $M$ & $S D$ & $M$ & $S D$ & $M$ & $S D$ & $M$ & $S D$ \\
\hline Surface form & $5.1^{*}$ & 1.0 & $5.2^{*}$ & 1.5 & $4.7^{*}$ & 1.1 & $5.0^{*}$ & 1.9 \\
\hline Textbase & $4.0^{*}$ & 1.8 & $3.9^{*}$ & 1.5 & $4.1^{*}$ & 1.7 & $5.7^{*}$ & 1.5 \\
\hline Situation model & 2.4 & 1.2 & $2.9^{*}$ & 1.2 & 2.3 & 1.0 & $5.2^{*}$ & 1.1 \\
\hline
\end{tabular}

Note-Maximum comprehension score $=8$. See text for explanation of conditions (NT-NT, NT-T). $\quad{ }^{*} p<.05$ (indicates mean is greater than chance). 
or textbase $(\mu=3.90)$ questions. On the other hand, during the second NT-T reading, accuracies for surface form $(\mu=5.00)$, textbase $(\mu=5.65)$, and situation model $(\mu=$ $5.20)$ questions were very similar. This suggests that readers' representations during the second reading of NT-NT reflected mostly surface and textbase knowledge, whereas readers' mental representations after the second reading of NT-T reflected all levels of comprehension.

Experiment 3 replicates the basic findings from Experiments 1 and 2 and further validates the comprehension questions. It also replicates the title effect found in Experiments 1 and 2 in a more controlled setting. Experiment 3 also demonstrates that the questions do not give away the topic of a passage. Only when a title is added during the second reading does situation model comprehension increase substantially.

\section{GENERAL DISCUSSION}

The results from the Experiments 1 and 2 suggest that (1) the presence/absence of a title mainly influences the situation model, (2) the questions are valid measures of the levels of comprehension, and (3) the rate of answering situation model questions correctly is not dependent on the amount of cognitive resources available. Experiment 3 showed that accuracy rates for situation model questions fluctuate as a function of presence/absence of a title but not of rereading, which also shows that the questions do not provide hints about the topics of the passages. The experiments described here also demonstrate critical issues that need to be considered when developing these types of passages and questions. For example, questions cannot overlap in textual content or contain cues that help the reader understand the topic of a text.

There are many uses for these types of passages and quizzes. For example, a researcher might be interested in studying the forgetting rate for the surface form and the textbase with and without a well-developed situation model. This could be done by asking people to read and recall the texts after various delays and with or without titles. Multiple-choice questions could be used to study this issue, but other measures, such as free recall, also would be appropriate. One difficulty associated with free recall is that the recall protocols must be scored. This is often done by counting the number of propositions, or idea units, recalled. To facilitate this type of research, a propositional analysis of each passage was conducted using the technique reported by Boviar and Keiras (1985). A sample for the text "Traditionally Mailing a Letter Versus E-mailing" is provided in Appendix B. Complete propositional analyses for each passage can be obtained by contacting the authors. The propositional analyses can be used to determine which ideas units are recalled. For example, if a reader recalled "How we perform the activity has been altered," credit for Propositions 1, 2, and 3 would be given for Sentence 1.

These passages and questions could also be used to study development. For example, children focus more attention on decoding words than on the overall meaning of a passage (Faulkner \& Levy, 1999). Conversely, older adults focus more on the gist (situation model) of the passage than on individual words (Stine-Morrow, Gagne, Morrow, \& DeWall, 2004). Our passages could be used to compare the rate of answering each type of question across age groups (i.e., children and adults) or with each age group. Within each age group, we would expect a smaller title effect for beginning readers than for highly skilled readers. For example, we would expect individuals who are learning English as a second language to show a smaller title effect than would fluent English speakers. Perhaps these passages could be used in some manner as a test of second-language comprehension.

There are several other methods for disentangling the readers' mental representation through the use of text manipulations. For example, Deane, Sheehan, Sabatini, Futagi, and Kostin (2006) explored how computational methods could be used to identify variation in text difficulty within texts across grade levels. They found systematic difference in lower-level features, such as word familiarity, and higher-level features, such as whether oppositional reasoning is used. To draw a comparison with our study, the purpose of our no-title condition was to minimize the abilities to form coherent linkages between pieces of information in a text and to develop a situation model. Deane et al.'s method can be used to specify what types of factors make situation-level processing differentially difficult across normal texts.

Gorin (2005) explored the influence of text features on question answering. Gorin found that questions with negative wording and passive voice were harder to answer correctly than semantically comparable questions. Questions were designed to tap comprehension of the textbase, which suggests that the textbase is influenced by negative wording. In contrast, modifying the propositional density and presentation of information in questions did not alter accuracy. Gorin's research provides insight into text manipulations that hinder comprehension. The similarity to our study is clear-Gorin manipulated wording and tested how this influenced the representation and comprehension of text. We manipulated comprehension difficulty through the presence or absence of a title and directly assessed comprehension of the surface form, textbase, and situation model.

In summary, we validated the title effect by creating comprehension questions that measure memory for the surface form, textbase, and situation model. We isolated the situation model as the main source of difficulty by showing that when a title is present, the accuracy rate for answering situation model questions is high, whereas when a title is absent, the accuracy rate for answering situation model questions is low. Accuracy rates for answering surface form and textbase questions were less influenced by the presence or absence of a title. These passages and quizzes provide researchers with a well-controlled tool for studying memory and comprehension.

\section{AUTHOR NOTE}

We thank Betty Ann Levy for allowing us to use the passages she developed. Correspondence concerning this article should be addressed to F. Daniel or G. E. Raney, University of Illinois at Chicago, Behavioral Sciences Building, Mail Code 285, 1007 West Harrison Street, Chicago, IL 60607 (e-mail: fdanie2@uic.edu or geraney@uic.edu). 


\section{REFERENCES}

Boviar, S., \& KeIRas, D. E. (1985). A guide to propositional analysis of research on technical prose. In B. K. Britton \& J. B. Black (Eds.), Understanding expository text (pp. 315-362). Hillsdale, NJ: Erlbaum.

Bransford, J. D., \& Johnson, M. K. (1972). Contextual prerequisites for understanding: Some investigations of comprehension and recall. Journal of Verbal Learning \& Verbal Behavior, 11, 717-726.

Deane, P., Sheehan, K. M., Sabatini, J., Futagi, Y., \& Kostin, I. (2006). Differences in text structure and its implications for assessment of struggling readers. Scientific Studies of Reading, 10, 257-275.

Dunlosky, J., \& RaWson, K. A. (2005). Why does rereading improve metacomprehension accuracy? Evaluating the levels-of-disruption hypothesis for the rereading effect. Discourse Processes, 40, 37-55.

FAUlKNeR, H. J., \& LeVy, B. A. (1999). Fluent and nonfluent forms of transfer in reading: Words and their message. Psychonomic Bulletin \& Review, 6, 111-116.

Gorin, J. S. (2005). Manipulating processing difficulty of reading comprehension questions: The feasibility of verbal item generation. Journal of Educational Measurement, 42, 351-373.

Haenggi, D., \& Perfetti, C. A. (1992). Individual differences in reprocessing of text. Journal of Educational Psychology, 84, 182-192.

KinTsCH, W. (1994). Text comprehension, memory, and learning. American Psychologist, 49, 294-303.

Kintsch, W., Welsch, D., Schmalhofer, F., \& Zimny, S. (1990). Sentence memory: A theoretical analysis. Journal of Memory \& Language, 29, 133-159.

LEVY, B. A. (2001). Text processing: Memory representations mediate fluent reading. In M. Naveh-Benjamin, M. Moscovitch, \& H. L. Roediger III (Eds.), Perspectives on human memory and cognitive aging: Essays in honour of Fergus Craik (pp. 83-98). New York: Wiley.

Levy, B. A., Campsall, J., Browne, J., Cooper, D., Waterhouse, C., \& Wilson, C. (1995). Reading fluency: Episodic integration across texts. Journal of Experimental Psychology: Learning, Memory, \& Cognition, 21, 1169-1185.

Levy, B. A., Di Persio, R., \& Hollingshead, A. (1992). Fluent rereading: Repetition, automaticity, and discrepancy. Journal of Experimental Psychology: Learning, Memory, \& Cognition, 18, 957-971.

McNamara, D. S., Kintsch, E., Songer, N. B., \& Kintsch, W. (1996). Are good texts always better? Interactions of text coherence, background knowledge, and levels of understanding in learning from text. Cognition \& Instruction, 14, 1-43.

Millis, K. K., Simon, S., \& TEnBroeK, N. S. (1998). Resource allocation during the rereading of scientific texts. Memory \& Cognition, 26, 232-246.

RANEY, G. E. (1993). Monitoring changes in cognitive load during reading: An event-related brain potential and reaction time analysis. Journal of Experimental Psychology: Learning, Memory, \& Cognition, 19, 51-69.

RANEy, G. E. (2003). A context-dependent representation model for explaining text repetition effects. Psychonomic Bulletin \& Review, 10, 15-28.

Raney, G. E., Therriault, D. J., \& Minkoff, S. R. B. (2000). Repetition effects from paraphrased text: Evidence for an integrated representation model of text representation. Discourse Processes, 29, 61-81.

Stine-Morrow, E. A. L., Gagne, D. D., Morrow, D. G., \& DeWall, B. H. (2004). Age differences in rereading. Memory \& Cognition, 32, 696-710.

VAN DiJK, T. A., \& Kintsch, W. (1983). Strategies of discourse comprehension. New York: Academic Press.

WiLEY, J., \& RAYNER, K. (2000). Effects of titles on the processing of texts and lexically ambiguous words: Evidence from eye movements. Memory \& Cognition, 28, 1011-1021.

Zwann, R. A., Magliano, J. P., \& Graesser, A. C. (1995). Dimensions of situation model construction in narrative comprehension. Journal of Experimental Psychology: Learning, Memory, \& Cognition, 21, 386-397.

\title{
APPENDIX A
}

Sample Text, Comprehension Questions, and Accuracy Rates for Experiments 1 and 2

\author{
Traditionally Mailing a Letter Versus E-mailing
}

In recent years the manner in which we engage in this activity has been altered drastically. Some people argue that with the advent of modern technology the entire process has been absolutely transformed. While both the traditional and the contemporary methods remain in use today, it is worried that the conventional process will soon be abandoned entirely. Indeed, while the elderly are most familiar with the traditional method, young people are often more comfortable with the modern approach. Further, the conventional process has been described as an "art" in itself. As children today are rarely taught the nuances and subtleties of this art, fears that tradition will be drowned by technology may soon be realized. Lately, a debate has arisen over the positive versus negative aspects of both the traditional as well as the contemporary approaches to this task. Let us consider the pros and cons of both of these methods. First, the tools appropriate for the activity differ according to method. The tools used in the conventional approach, for example, are readily accessible and cheap to buy; however, a small additional fee must be paid each time the task is completed. In contrast, the modern approach involves an initial investment in expensive equipment with no additional fee for each transaction. With respect to time, additional differences are noted between the traditional and contemporary methods. Still, these differences do not apply to the entire process. Most individuals require the same amount of time to complete the majority of the task using either method. The modern approach is merely faster in the last stage of the process. It is at this stage that the responsibility for completing the task is taken out of the hands of the participant and is instead managed by an outside source. Notably, the time difference between methods in this latter portion of the process is very significant. It may take hundreds of times as long to complete the task using the traditional as compared to the modern method. Further, it is on this basis that most individuals argue in favor of universally adopting the latest approach. Given the increased emphasis on time-management today it is not surprising that individuals value the efficiency of the contemporary method. Still, traditionalists believe that one's style and personal touch can only be portrayed using conventional means, and that these characteristics far outweigh any time advantages the modern approach may provide. 


\section{APPENDIX A (Continued)}

\begin{tabular}{lll}
$\begin{array}{l}\text { Experiment 1 } \\
\text { No Look Back }\end{array}$ & & \multicolumn{2}{c}{$\begin{array}{c}\text { Experiment 2 } \\
\text { Look Back }\end{array}$} \\
\cline { 3 - 4 } $\mathrm{T}$ & $\mathrm{TT}$ & $\mathrm{T}$
\end{tabular}

\section{Surface Form}

(1) Which word was in the original sentence?

58

51

100

81

While both the traditional and the contemporary methods remain

in use today, it is worried that the conventional will soon

be abandoned entirely.
(a) means
*(b) process
(c) ways
(d) approaches

(2) Which word was in the original sentence?

Further, it is on this basis that most individuals argue in favor of universally the latest approach.
(a) accepting
(b) securing
(c) acquiring
*(d) adopting

Textbase

(1) Which is a disadvantage to the modern approach?

(a) It requires a fee each time the process is completed.

*(b) There is a required investment in expensive equipment.

(c) It is hard to find tools needed for this approach.

(d) This approach is less familiar to all people.

(2) Which part of both approaches to this activity is virtually the same?

(a) The planning required to complete the task.

(b) The tools we use to perform the activity.

(c) The time-management involved after the activity is performed.

*(d) The time required to perform the majority of the task.

$\begin{array}{lllll}\text { Mean } \quad 59 & 61 & 100 & 85\end{array}$

45

79

\section{Situation Model}

(1) When using the traditional approach, what is required for each transaction?

(a) An investment in expensive equipment.

*(b) An adhesive picture that is placed on the final product.

(c) A line that indicates the content of the final product.

(d) A push of a button that allows you to create the final product.

(2) What is an advantage that is experienced by the receiver of the modern approach?

(a) The contents of the final product are more thorough.

(b) The final product is more organized.

(c) The final product is more aesthetically pleasing.

*(d) The final product can come more than once a day.

$\begin{array}{lllll}\text { Mean } & 52 & 45 & 83 & 63\end{array}$

$\begin{array}{llll}66 & 23 & 64 & 50\end{array}$

\begin{tabular}{lllll} 
Mean & 64 & 33 & 64 & 38 \\
\hline
\end{tabular}

Note-T, title presented; NT, no title presented. 


\section{APPENDIX B}

Propositional Analysis for Sentences 1-5 From "Traditionally Mailing a Letter Versus E-mailing"

1. In recent years the manner in which we engage in this activity has been altered drastically.

P1(ALTERED WE MANNER)

P2(ENGAGE-IN WE IN-ACTIVITY)

P3(IN-WHICH P1 P2)

P4(TIME P1 IN-RECENT-YEARS)

P5(MOD P1 DRASTICALLY)

2. Some people argue that with the advent of modern technology the entire process has been absolutely transformed.

P1(ARGUE PEOPLE P2)

P2(TRANSFORMED TECHNOLOGY PROCESS)

P3(AMOUNT-OF PEOPLE SOME)

P4(MOD TECHNOLOGY ADVENT-OF)

P5(MOD TECHNOLOGY MODERN)

P6(PART-OF PROCESS ENTIRE)

P7(MOD P2 ABSOLUTELY)

3. While both the traditional and the contemporary methods remain in use today, it is worried that the conventional process will soon be abandoned entirely.

P1(REMAIN-IN-USE TRADITIONAL-METHODS \$)

P2(REMAIN-IN-USE CONTEMPORARY-METHODS \$)

P3(TIME P1\&P2 TODAY)

P4(WORRY \$ P7)

P5(ABANDON \$ CONVENTIONAL-PROCESS)

P6(MOD P5 ENTIRELY)

P7(TIME P5 SOON)

P8(WHILE P3 P4)

4. Indeed, while the elderly are most familiar with the traditional method, young people are often more comfortable with the modern approach.

P1(ISA ELDERLY FAMILIAR)

P2(WITH P1 TRADITIONAL-METHOD)

P3(ISA YOUNG-PEOPLE COMFORTABLE)

P4(WITH P3 MODERN-APPROACH)

P5(AMOUNT-OF P3 OFTEN)

P6(MOD FAMILIAR MOST)

P7(MOD COMFORTABLE MORE)

P8(WHILE P2 P5)

P9(INDEED S3:P4 S4:P6)

5. Further, the conventional process has been described as an "art" in itself.

P1(DESCRIBE CONVENTIONAL-PROCESS AS-ART)

P2(MOD ART IN-ITSELF)

P3(FURTHER S3:P4 S5:P1)

(Manuscript received August 29, 2006;

revision accepted for publication December 27, 2006.) 